\title{
Newsbots that mediate journalist and audience relationships
}

\begin{abstract}
News media organisations are experimenting with a new generation of newsbots that move beyond automated headline delivery to the delivery of news according to a conversational format within the context of private messaging services. To build the newsbot, journalists craft statements and answers to users' questions that mimic a natural conversation between a journalist and user. In so doing, journalists are experimenting with styles of communication that reflect very particular journalistic personas. We investigate the persona of the news chatbot created by the Australian Broadcasting Corporation (ABC), the better to understand how the public broadcaster's forays into social media service delivery and automation are shaping new relationships between public service broadcasters and their audiences. We find that, for the audience that uses it, the friendly newsbot contrasts with everything they previously thought journalists to be. The public service journalists who operate the bot are, in turn, using the bot to try to reach new audiences by experimenting with a more informal, intimate relationship with citizen users. The supposedly "intelligent" (but in actual fact very much human-crafted) newsbot is the vehicle through which this new relationship is being forged.
\end{abstract}

\section{Keywords}

Newsbots; automated media; conversational journalism; public service media; algorithmic journalism

\section{Introduction}

Bots are software applications that perform automated tasks over the internet. Journalists have used bots for tasks relating to both the production and dissemination of news, with the term "newsbots" typically applied to applications that help journalists (and sometimes their followers or readers) keep track of particular topics or stories by extracting news headlines automatically from other news sources (Lokot and Diakopoulos 2015). When newsbots have interacted with readers on social media platforms such as Twitter and Facebook, they have tended to function predominantly as rebroadcasters of traditional news content to social media (Lokot and Diakopoulos 2015, 694).

A more recent development, however, has seen the spread of newsbots from public social media platforms to private messaging services such as Facebook Messenger. This development results in newsbots incorporating features of chatbots in addition to functioning as disseminators of news. Chatbots are computer programs that respond to user input by simulating how a human would behave as a partner in a conversation. According to Bradshaw (2016), the first wave of bots in journalism saw early Twitter bots being used by journalists for "alerting, aggregating, and monitoring", whereas chatbots are now being developed with the ultimate goal of conversing directly with the user.

Technological advancements have also been propelled by changes in user practices, both by the move to private messaging channels (and away from social media's public channels) and an increasing acceptance by users of conversational interfaces. Since 2015 users of messaging apps (including WhatsApp, Facebook Messenger, WeChat and Viber) have outstripped active users of the most popular social networks (BI Intelligence, quoted in Newman et al. 2017, 15). According to Newman et al. $(2017,17)$, this is because "people increasingly prefer to share content not on big open networks... but within apps like WhatsApp, 
Snapchat and Facebook Messenger where they can have more control". This trend has continued, as evidenced in the 2018 Reuters Digital News Report, which describes an increase in news consumption via mobile and the growing use of voice-activated speakers (such as Amazon Echo and Google Home) to access news (Newman et al. 2018). Asking your smart speaker for the latest news may therefore become a significant way of receiving news in the future.

In this paper we investigate the changing relations between media organisations and audiences enabled by this new generation of newsbot by considering the newsbot of the Australian Broadcasting Corporation (ABC), which was launched in 2016. The ABC is Australia's national public broadcaster. Founded in 1932, it is principally funded by the Australian government, but maintains a set of principles that ensure its independence from government. These principles include public service commitments to universality, excellence, diversity, accountability, and innovation (Tracey 1998).

Each of the public media principles is subject to reconfiguration with the shift from public service broadcasting (PSB) towards public service media (PSM) in the context of an increasingly networked environment. The role of PSB now includes not only producing and procuring content for its traditional television and radio offerings, but also designing and developing digital information and distribution services that align with an ever-splintering public that has specific and niche demands (McClean 2011). This shift to PSM, alongside the legislated requirement to innovate, situates public media not only at the coal-face of technological development, but also as an ideal institution to experiment with and integrate a number of human-machine communication approaches.

Such developments inevitably impact on the principles underlying public service media organisations. Van de Bulck and Moe $(2017,16)$ demonstrate how the majority of such organisations in Europe are moving towards algorithmic personalisation which they see "as a tool to realise universality in new ways". Van Dijck and Poell $(2015,148)$, looking at PSMs and their integration into social media, note that when "the boundaries between public and corporate online space are becoming progressively porous, the meaning of 'publicness' is contested and reshaped".

There are diverging opinions about whether these changes are positive or negative. Some contend that increasing pressures to adopt personalisation technologies or to work with third-party (commercial) social media players to deliver services have a negative effect on issues such as universality, diversity and accountability (see, e.g., Born 2005). Others (including Flew 2011; Debrett 2010; Jakubowicz 2007) are optimistic about the changes, arguing that public media organisations are innovating with new modes of delivery that enable them to reach diverse publics across multiple platforms, thereby complementing, rather than undermining, the principle of universality.

Beyond these scholarly debates, an alternative path towards understanding how new technologies influence the values and principles underlying public service media involves investigating how those technologies mediate new relationships between the PSM organisation and the citizens they serve. In doing so, we move from framing newsbots as a medium with which we have a relationship towards framing them as a medium that mediates existing social relationships (in this case between audiences and PSM organisations).

By investigating the case of the $\mathrm{ABC}$ news chatbot, we offer insights into the opportunities and challenges that such technologies create for media organisations, particularly public service media organisations. After situating our research questions in the context of relevant theory and empirical research and describing our methods, we introduce the ABC's newsbot, beginning with a description of what the bot does, situating this within the context of the $\mathrm{ABC}$ and highlighting the ways in which its designers talk about it. We then move on to the newsbot's reception, outlining three key aspects of the newsbot's personality as identified by a selection of its audience. The article concludes with an analysis of what the bot's personality (as designed by journalists and received by users) tells us about the relationship between public service media organisations and the publics they serve. 
We argue that the move from using newsbots on public social media channels such as Twitter to using them on private messaging channels such as Facebook Messenger has resulted in key changes that demand a redefinition of newsbots for journalism studies. The changes are twofold. First, the emphasis in providing news by newsbot is not on an area of coverage (a topic, beat, or location of interest) but on the method of coverage, in this case according to temporal conditions (at a time specified by the user) and applying a conversational form of news delivery. Changes to the method of coverage result in key changes to journalistic practice.

More importantly, however, the move also represents an important shift in the relationship between journalists and citizens as the journalistic organisation moves into the private, conversational realm of messaging services. This shift requires a redefinition of the role, function and possibilities of newsbots.

\section{Literature review}

Research investigating the ways in which automated processes influence the news and news media organisations is preceded by a long history in the field of human-computer interaction, where research has investigated how humans receive information delivered via automated processes or sources. In their experimental research on computer personality, for example, Moon and Nass (1996) found that it was possible to operationalise a computer's personality using "a rudimentary, text-based system with scripted responses" (668) and that their research participants recognised computer personalities as "psychologically real" (651). Finding that people preferred different types of personality that corresponded with their own, they concluded that "attempts to design a single computer personality that appeals to all users are (therefore) misguided" (668).

If people recognise the personality traits programmed into a technology and act towards that technology as if it were a human, the relevant question for journalists is what types of characteristic are deemed most credible? Sundar and Nass (2001) asked research participants to rate the credibility, likeability, quality and representativeness of online news stories as a function of different types of communication sources. They found that when either the computer terminal or other audience members were perceived to be the source of news, the stories were rated higher in quality than when news editors were perceived to be the source (65).

Recent studies have reinforced this earlier work, pointing towards trustworthiness that users ascribe to third-party, computerised actors and algorithmic processes used for selecting and curating the news. Thurman et al. (2018), for example, demonstrate a link between respondents' trust in news organisations and their assessment of the utility of algorithmic and editorial news selection. They found that as trust in news organisations falls, people are less likely to recognise news selection by editors and journalists as a good way to get news. By contrast, agreement that automated personalisation is a good way to receive news is less affected by distrust in the media. They concluded that users do not recognise the link between automated news personalisation and the operation of news organisations, "believing the technology has a degree of immunity from contamination by a politically compromised or untrustworthy news media" (17).

Despite these trends, there are still major gaps in our understanding of trustworthiness and credibility in relation to automation. The Reuters Digital News Report (Newman et al. 2018), for example, reports that $65 \%$ of people surveyed from 40 countries said they prefer to get news via search engines, social media or news aggregators (this figure goes up to $73 \%$ for under 35s). But when asked what sources of news they trust, only $34 \%$ trust search engines and $23 \%$ trust news from social media most of the time.

In order to understand the consequences for trustworthiness in the case of newsbots, we extend the research in this area by focusing on a particular case. We frame the study not in terms of newsbots as a medium with which we have a relationship, but in terms of newsbots as a medium that mediates existing social relationships (in this case between audiences and media organisations). Here, we follow Höflich (2013) who proposes a triadic model of human-robot 
interaction rather than the traditional dyadic model, and suggests that robots can be either a connective or divisive element in the inter-group relationships that they mediate.

Writing about the role of social robots in the context of interpersonal relations, Höflich explores the idea of social robots as media. Instead of seeing a social robot as a machine with which humans interact, Höflich argues that robots need to be recognised both a medium with which humans interact but also as a medium that mediates. This triadic analysis of mediaoriented behaviour recognises the robot as a third party in interpersonal relations that affects how we see ourselves and how we relate to others in a relational context. Höflich explains that the robot as third party can either connect or separate the persons whose relationship it mediates.

We apply Höflich's framework to the case of newsbots within a larger mass communication context. The newsbot, in this case, is recognised as a third entity that presents the frame of communication between audiences and PSM organisations, and is also framed (or recognised) on its own (43). This relational perspective enables us a glimpse into the meaning of the newsbot in context, i.e. as a third party that can either connect or separate. The question in this case, then, becomes whether the newsbot connects audiences to their public service broadcaster, or separates them from it, and what role automation plays in this relationship.

\section{Method}

Our method employed several ethnographically inspired techniques and practices (Pink et al. 2015). The goal of the project was to explore not just what the newsbot did, but who the bot was for readers and journalists, and for the PSM organisation that constructed it, and then explore the connections between them. We adopted Nagy and Neff's $(2015,1)$ framing of human-computer relations that includes "users' perceptions, attitudes, and expectations; the materiality and functionality of technologies; and the intentions and perceptions of designers" (themes that we have used to structure our findings). Following Guzman $(2017,76)$, our tracing of relations focused on "both what others say about (the bot) and what it says about itself (verbally and nonverbally), keeping in mind the cultural contexts that surround these elements".

We implemented a mixed methodology that draws on what Seaver (2017) calls the "scavenger" approach, which was particularly helpful in gleaning information from diverse sources. As Seaver notes, "The scavenger replicates the partiality of ordinary conditions of knowing - everyone is figuring out their world by piecing together heterogeneous clues - but expands on them by tracing cultural practices across multiple locations and through loosely connected networks" (6). In implementing this method, we combined data from interviews, surveys, field diaries, policy documents and our own experience of using the newsbot to expose the triadic relationship between the three key stakeholders.

We interviewed via phone and email the managers and designers behind the $\mathrm{ABC}$ newsbot. The team is located in Brisbane, Queensland and is responsible for the technological development of the News Division around the country.

Those same ABC employees provided access to user survey data and user responses to the bot including the results from an August 2017 survey of 3404 newsbot users, a selection of 120 substantive anonymous user responses about the bot (from 8000 pieces of feedback that included topic suggestions, technical issues and emojis), and 101 responses from users about why they uninstalled the bot, as well as internal presentations of user feedback and survey responses. The policy documents we examined were a mix of ABC public and internal strategy documents, including the $\mathrm{ABC}$ Annual Reports, legislated Acts and external-facing advertising material.

We also recorded our own field notes when we installed and used the bot for a period of over six months (Light, Burgess, and Duguay 2018). During this time, we took screenshots and then annotated them according to surprises, reflections and iterative interpretations. Tracing a divergent set of data points that had been scavenged from a number of sources helped us to 
identify and understand the media assemblage surrounding the newsbot: a media assemblage of corporate technology providers, the public service media institution, journalists, technologists, and the public, along with non-human actors including the newsbot and the technologies that are summoned in justifying the new service.

In the following three sections, we analyse the ABC's news newsbot according to its materiality and functionality, the intentions and perceptions of its designers and newsbot users' attitudes towards, and expectations of, the bot.

\section{The materiality and functionality of the bot}

"G'day Jono, welcome to ABC News on Facebook Messenger, your Australian news assistant. We're here to get you up-to-speed ASAP whenever you need us, with morning and evening news summaries, breaking news alerts and more.

We can also tell you when major news breaks, so you can finally conquer your FOMO.

Don't worry, this will only be for the really big stuff. And the best bit is we won't just tell you WHAT has happened, we'll tell you WHY it's important.

Are you in?"

This was the response that greeted us after we searched for ABC News on Facebook and clicked on the "Message" button to start a conversation with the ABC newsbot. We selected "I'm in" and then moved through the news topics that we wanted to follow (including politics, world politics, entertainment, business, sport, announcements and quiz). The newsbot asked whether we wanted to subscribe to breaking news in addition to daily summaries of the topics, and we were able to select a time to receive the news summaries.

Interacting with the newsbot, we saw that daily news summaries include three stories of interest: an article of high public interest, an article of local significance, and a "feel good" story. At each point the user is presented with a list of options for responding to the bot, such as "tell me more", "why did this happen?" and "what does this mean?". While this is the standard approach of the $\mathrm{ABC}$ newsbot, the user is also presented with the option to delve deeper into surrounding news items through the "more like this" option.

In terms of newsbot functionality, then, the newsbot delivers automated news alerts at a time specified by the user, is also able to deliver targeted news according to topics that the user is interested in, to register responses from the user to individual message elements (using Facebook's "reactions" including thumbs up, thumbs down etc) and to answer questions composed by the user.

For example, we asked the bot:

"What's your name?"

To which it answered:

"My name is $A B C$ newsbot. Because I'm a newsbot... from the ABC.

That's a clever name, right?"

During important national events, the bot might be taken over by a special reporter who gives a personal take on what is happening, why it is important and what readers should know about it. During the May 2017 federal budget announcement, the chatbot was operated by ABC journalist Peter Marsh who used his personal experience "as a political nerd heading into a 
budget lockup for the first time" to give readers "an inside look at how it all happens" (Marsh 2017). The bot introduced Marsh to audiences and provided updates throughout the night's proceedings. According to the journalist,

There was a mix of actual news from the budget and lighter personal commentary from me and we weren't afraid to get playful with the tone, considering the audience. (Craig McCosker, personal communication, 9 October, 2018)

As previously stated, the chatbot typically delivers three headlines to the user in a daily news summary delivered via Facebook Messenger (see Figure 1 below).

\section{[Insert Figure 1 near here]}

Figure 1. An example of an $\mathrm{ABC}$ newsbot morning news alert

The bot is structured around a question and answer format. Newsbot users can receive headlines and choose to "tap through for more" information based on these headlines. Each snippet contains an image followed by two or three sentences highlighting the key points of the story. Users can then ask for more information about the story when delivered a prompt such as "tell me more" or they can "move onto the next story". Alternatively, prompts may be more specific to the context of the story reflecting questions that might pique the user's curiosity.

The example below from 12 September 2017 demonstrates how a story might unfold on the newsbot:

The United Nations Security Council has imposed new sanctions on North Korea in response to last week's nuclear test.

But they're not as tough as the US wanted.

Users are then prompted with the question "How so?" When this option is clicked, the story evolves:

America wanted an oil embargo, but instead crude oil exports to North Korea will be capped at current levels.

The new sanctions also ban textile exports from North Korea and prohibit all countries from authorising new work permits for North Koreans.

US envoy to the UN Nikki Haley said North Korea had 'not yet passed the point of no return'."

Users can then either "read the story" on the ABC's website or move on to the "next story". In addition to daily news and news alerts being delivered in this interactive format, users are also able to ask a question or send a message to the bot.

Testing the functionality of free text questions to the bot, we realised that its ability to respond to questions is limited. For example, when we asked the bot "Who is Justin Timberlake?" after a message delivering a headline about Timberlake at the Super Bowl, the bot responded with "I can do a search of the news for you if you like?" (a typical answer to queries of this type).

When we provided the bot with its requested keywords (in this case: "Justin Timberlake"), it returned a story about Timberlake from that morning's news, rather than an answer to our question. Although the newsbot will respond to questions about what the latest 
news is, it was not able to answer our question about what was trending on social media (see Figure 2 below).

\author{
[Insert Figure 2 here]
}

Figure 2. The ABC newsbot's response to our question: "What is trending on social media?"

Although the bot simulates a human conversation in terms of the placement of its modular story segments, all the user response options are decided by the news creators. If users have questions other than "And then what happened?" for example, the conversation will reach a dead end. Users can respond to the bot's statements with Facebook's "reactions", but cannot comment on the news in the way that they might be able to with news articles on a news site. It is unlikely that users, when choosing from different options, will believe they are engaging with a human rather than a bot, as the responses to user input are instantaneous and free text questions to the bot are so limited. Users may, however, question whether their responses to specific requests for feedback or to individual story elements using Facebook's carousel of reactions are being read and listened to by journalists at the ABC.

\title{
The intentions and perceptions of designers
}

The content for the bot (including daily news summaries, breaking news and answers to free text user queries) is developed by the ABC News Mobile team, located in the News Technology Division in Brisbane. The team is also responsible for developing content for the ABC's other mobile news products. While the majority of the News broadcast comes out of the Sydney studios, a significant amount of the news technology is developed and deployed from the Brisbane location.

The newsbot was built using ChatFuel, a Web-based platform. Founded in 2015 by Russian entrepreneurs Dmitrii Dumik and Artem Ptashnik and based in Silicon Valley, ChatFuel is primarily focused on enabling non-developers to build bots within Facebook's Messenger service. The company offers free services and also subscription services that remove ChatFuel branding and provide access to more fine-tuned data about the audience. While the ABC started with the free version, they have since moved to the premium version to enable greater personalisation.

Craig McCosker, product manager for ABC News Mobile team, led the development of the newsbot. When we asked about the development process for the bot, McCosker talked about the team's experimentation with the available technology, with topic selection, news story explanations and the personality characteristics of the bot. According to McCosker, the conversational, guided, interactive approach to designing newsbots in the messaging context requires much more effort than the automatic delivery of stories with standard headlines and descriptions.

One of the greatest challenges for news producers developing newsbots in the conversational format is the personality of the bot. A chatbot's personality is developed by its creators through the tone and character of the answers provided to users' questions (Verma 2018). Enabling the bot to respond to a wide variety of free text responses from users through Artificial Intelligence applications (at the level of the application) and delivery (at the level of content production) is a key challenge. The challenges are not only technical, however. Newsbot designers developing the chat facilities in messaging environments must also develop a style of conversation that is both authoritative and personable.

The question that bot designers and developers are asking is: "What kind of human will the bot be?" This applies not only to the ways in which the bot "reads the news" - or, in this case, delivers and frames the news - but to the tone with which the bot responds to user queries. Making the bot sound "human" is a challenge for journalists, as is the fact that the 
colloquial nature of conversation is often at odds with the goals of authority and credibility that may result in news products of a very different tone.

According to the $\mathrm{ABC}$ team, newsbot messages need to feel personal (rather than "robotic"), but they were initially uncertain about "how far to go regarding the creepiness of personalisation" by addressing users by name. However:

"[W]e tested it and overall people found it more engaging and/or got used to it after a short time." (Craig McCosker, personal communication, 9 October, 2018)

The style and tone of the $\mathrm{ABC}$ newsbot has been the target of continuous readjustment. The team imagines the newsbot as "a smart, newsy friend in your pocket". This phrase highlights both the personality characteristics of the newsbot and the relationship the team is working towards in the development of conversations with users.

In relation to personality characteristics, the team initially tried signalling the automated nature of the newsbot using icons, but abandoned the "friendly robot icon" and the "news robot style personality", because they felt that this was "constraining and unnecessary" (McCosker interview, 9 October, 2018). The style of conversation has been more difficult to refine, as the $\mathrm{ABC}$ news team continuously tries to achieve a balance that makes the newsbot acceptable to a wide range of audiences. McCosker reported that while some liked the conversational style that involves being guided through the news in dialogue form, others wanted the more traditional approach that enabled them to immediately view all the headlines from which they could select individual stories.

"[While] many people [were] keen to engage in a conversational style of news... others found it too slow and [an] unnecessary overhead compared to... scanning lots of headlines on [a] website or app homepages. The conversational, emoji, background and shared experiences-rich style that resonated with many young people was a turn off for older people who wanted to use the bot but found the style used to talk about each story trivial, condescending and not serious enough - just the facts please. The style used is now more middle of the road and is accessible for more people but it's an open question for research [whether we] have we lost an edge with a younger audience." (Craig McCosker, personal communication, 9 October, 2018)

The prospect of attracting this younger audience (along with other previous "non-users" of ABC news products) is what most excited the news team about the newsbot's development. Since its launch in 2016, the user base has grown to about 600,000. That audience, according to the chatbot news team, is younger (66\% of users surveyed in the latest poll were under 45 ) and more likely to be female (55\%) than the typical ABC News audience, reflecting general trends in social media demographics (Tolliday 2017). This is an audience that the chatbot team recognises is traditionally underserved by ABC News. One of the core aims of the newsbot, for the team, has been making news more accessible to these previous non-users.

"It's about lowering barriers around background knowledge and [increasing] motivation to [engage] with the news... making it accessible to people with lower levels of education and interest in current affairs." (Craig McCosker, personal communication, 23 March, 2018)

Another aspect of the relationship with users that the ABC newsbot team is aiming for is reflected in their conception of the newsbot as a "smart friend in your pocket". The intimacy of the term "friend" reflects the personal, conversational design of the service and also the particularly trusted role that the $\mathrm{ABC}$ sees for itself. According to McCosker, "The ABC is very trusted. We see ourselves as a guide or companion via a mobile that is with you all day... [and is] trusted to reach out to you and push-alert you with news." In McCosker's view, the ABC newsbot 
provides an "essential, informative, concise and approachable" take on news media, inviting the audience to participate in a personalised news experience.

The team regularly receives information about their users' responses and feedback to individual stories and the service as a whole. McCosker views this constant feedback as a means of cultivating a closer relationship between the $\mathrm{ABC}$ and their audiences than is provided by other services on offer.

"We have also been increasingly doing calls out to the Messenger audience to share their experiences and perspectives to build out our newsgathering and make storytelling richer. I see all of this as an attempt to make a news service distinctive through having a closer relationship with audiences in contrast to the commodification of news content we see broadly on the mobile web." (Craig McCosker, personal communication, 9 October, 2018)

The $\mathrm{ABC}$ may see themselves as trusted providers of news, but what do users think of the newsbot? Is it as credible and trustworthy as the ABC claims? In the next section we highlight three traits of the newsbot's personality as experienced by some of its users.

\section{Newsbot users' attitudes and expectations}

Respondents to the ABC's 2017 survey (see "Method" above for more detail) were generally non-users of traditional news products and remarked that being informed of the news using the bot made them feel more empowered and more knowledgeable: they "love[d] knowing what is going on in the world so quickly and easily" (A5 $\left.{ }^{1}\right)$. Newsbot users also seemed to constitute an audience previously disenchanted with the news and the journalists who author it. In their responses about the bot from the 2017 survey (see "Method" above for more detail), the newsbot users who indicated that they were "by no means a lover of politics" (A2) or that they "don't watch the news or read the paper" (A1) or that they often "don't have the time to watch the news" (A7) - sometimes because of work, at other times because they were full-time carers - appreciated "having someone sending little updates" (A1) or "keeping [them] updated" (A2) or "having small bites of information [they] could access at any time" (A18).

Users who spoke positively about the newsbot often appreciated the personal nature of the communications. One user remarked:

I like the tone of all the $\mathrm{ABC}$ news messages. They feel professional yet personal. (A12)

Many of the positive comments mentioned the tone of the bot. Tone refers to the style in which the bot delivers the news, the attitude towards the subject expressed by the speaker, and the personality of the bot as shaped by the bot's creators. It is the result of writing style, the mix of textual and graphical elements, the ways in which the bot addresses the reader and the algorithms and data processes employed by the bot team to deliver news to the user.

In addition to asking what the bot does, it is important to understand the persona of the bot, how that persona is shaped by the bot's creators and how users respond to the bot. Who is the bot to the user? Many of the comments about the bot referred to characteristics normally understood as human traits. In the next section we highlight three traits of the ABC newsbot as experienced by its users. The bot is perceived by these users to be unpretentious, optimistic and helpful.

\section{Unpretentious}

Users expressed appreciation for the non-intimidating, non-threatening tone of the bot. User A1 remarked that the "news is presented in a fun, non-intimidating way and emojis and other 
quirky little additions just make the news that much more user friendly". Another wrote that the bot makes "reading the news a lot of fun" (A7).

News stories are fashioned using informal vocabulary native to social media. An emoticon waves at the user when they have finished reading the news, users can respond to stories with the same emoticons that Facebook enables in responses to posts on the site, and stories are almost always accompanied by some kind of graphic - either a photograph or animated gif with references to popular (internet) culture and internet memes. Accompanying a story from 7 February 2018 about the UK banning the purchase of bitcoins by credit card, for example, is an animated gif of a character from the Futurama animated comedy series with the words "Shut up and take my bitcoin" based on a popular internet meme ${ }^{2}$ from Reddit.

The style of writing, too, is irreverent and frames the news in original ways. A news alert from 8 February 2018, for example, covering Nancy Pelosi's eight-hour speech to the US Congress about the "Dreamers" - the immigrants brought to the US as children who now face deportation after President Trump threatened to remove their protection - begins with:

Someone please get Nancy Pelosi a drink of water!

This is followed by:

The Democratic leader just finished making an EPIC speech in the US House that went for a record-breaking 8 hours straight.

And it argued against a deal that would keep the government open...

The satirical opening to this story reflects $A B C$ journalists' wish to use an informal style associated with conversations between friends. If users click through the introductory snippet, they are able to navigate to the $\mathrm{ABC}$ website where the story is presented in full. These introductory snippets frame the story in ways that pique the user's curiosity by focusing on personalities and individuals' behaviours, before moving on to the policy issues. This accessible tone assures newsbot users that they too could find the news useful and interesting.

\section{Optimistic}

The bot is also perceived as being optimistic by a number of users surveyed, particularly because of the focus on good news. Users can sign up for a special news alert for "good news". The interface for selecting this option is framed as follows:

Tap 'I'm in' if you'd like us to send you a wrap-up of good news at noon each Wednesday. We think it'll give you that little bump of positivity you need to get through the day.

The bot typically starts the daily news message with two hard news stories (usually covering politics or major global headlines) and one feel-good story relating to entertainment, celebrity or technology. At the end of 2017 there was a special feature with a "list of 12 good news stories you might have missed while you were worrying about a nuclear war". One user responded to the good news angle:

Just love the ability to get the news that is current and is apt to my interests AND the good news stories not just the sad or terrible stories! (A64)

The creators have timed the delivery of the good news wrap-up for the mid-point of the week, when they think users might be most in need of a boost, and their general openness to the idea of positivity reflects a more intimate engagement with the needs of the bot users. 


\section{Helpful}

In addition to the bot being perceived as optimistic and non-intimidating, it is also perceived as useful. Many users commented positively on the "informative" nature of the bot. When the bot delivered a special feature on the Australian budget, one user stated:

I love these bots... and the budget, it is explained in a way that I understand. All the fluff taken out and simple to understand. (A22)

Two distinct categories of content are integrated in the bot's daily headlines. A news element focuses on telling the reader what has happened, whereas an information element directly assists the reader in decision-making. A story about the ABS's release of its latest Household Expenditure Survey, for example, indicated that "it isn't looking good for the average consumer" because "[w]e're spending more on the basics... than six years ago". If users chose the option of finding out how to cut down on household expenses, they were taken to a series of suggestions from experts about how to spend less.

A further example of the informative aspect can be found in the "News Explained" section of the newsbot. After running through the standard headlines, users are given the option of having the key news items of the day explained to them, and are taken to a broader "explainer" article that breaks the story into smaller descriptive texts (see Figure 3 below). This includes information on why the news item has emerged, what the key aspects of the news item are, and what the implications of the news item are. The audience is not only being exposed to the issues, but is having them explained in an accessible way.

[Insert Figure 3 near here]

Figure 3. Users are provided with an "Explainer" for the news headlines

\section{What the bot is not}

Users' remarks about what the newsbot is not are perhaps as interesting as what they say it is. Some users seemed to be saying that the bot, unlike journalists, is not pretentious, does not talk down at them, and is not always the bearer of bad news. Prior to their experience of news via the newsbot, the news for these users was depressing, intimidating and/or irrelevant. The bot, however, made one user "feel less overwhelmed when the morning/evening news arrives" (A15). It framed the news in ways that left some users feeling empowered, because they now knew what was going on in the world "quickly and easily" and in a way that avoided the negative effects of daily news that they had previously experienced and/or perceived. This enabled the bot to promise that the service would "conquer [the user's] FOMO" (fear of missing out), a message delivered when we first activated the bot, directly engaging with what the bot creators recognise as users' emotional needs.

These users respond to the bot because it frames the news for them in ways that are warm, welcoming and personal. One user noted that news items "feel professional yet personal" (A12). By the term "personal" they meant that the bot speaks to their personal/emotional needs, not that they think the bot is speaking to them personally in a way that will enable them to conduct a conversation with the bot.

For the younger public that makes up the bot's audience, then, the bot signals a new relationship with journalists and journalism. As one user commented:

"(I) feel like I can see some curly haired guy called Sid sitting in the ABC offices typing them out." (A12)

It is the personal tone of the bot, crafted by the journalists, that they appreciate and connect with. 


\section{A very human newsbot}

In this study we examined how an ostensibly automated product (the newsbot) is actually constructed through the careful work of humans - in this case journalists who carefully craft entry points into the news in ways that are experienced by users as unpretentious, optimistic and helpful by some users. The delivery of news appears to these users as just one part of a twoway conversation between themselves and the news organisation. Users of the bot respond in ways that reflect a new relationship with a media organisation that they might previously have rejected. The bot's utterances reflect a change in the tone of the news and in the persona of the journalist. This acceptance of the news chatbot (and the consequent credibility of its utterances) is achieved through the use of familiar, informal, colloquial language crafted as part of a conversation with individual users, rather than through formal announcements or proclamations.

Previous research on Twitter newsbots has defined newsbots as "automated accounts that participate in news and information dissemination on social networking platforms" (Lokot and Diakopoulos 2015, 683). However, the new generation of newsbots, as exemplified by the $\mathrm{ABC}$ newsbot, introduce fundamental changes to what newsbots might do and enable in the relationship between journalists and their audiences. Hoflich argues that robots should be seen in the context of a relationship with a medium but also as a medium that 'mediates'. In this case, newsbots are introduced in the context of social media messaging platforms using a conversational format. When the newsbot moves into the private, conversational and intimate space of social media messaging platforms (as opposed to the public spaces on these platforms) and adopts a particular persona, it becomes a significant third (person) mediating the relationship between audiences and their public service media organisation.

Changes are not only to the format of news produced for this audience; they are to the tone of the news and the authorial persona presenting it. The newsbots enable an intimate relationship between journalists and their audiences. For the journalists involved the bot offers a way to experiment with a new kind of informal, irreverent, conversational style of news and information sharing that represents an evolution of their relationship with their audiences. Journalists, in other words, are practicing a kind of ventriloquism with the bot, and some of the public participates in the ruse because it is entertaining and amusing, and, because the it is amusing, because news is told in a kind of style that they can relate to.

If the $\mathrm{ABC}$ newsbot works to connect the public service media organisation and this particular audience, how is this achieved? In the case of the ABC newsbot, we find that new connections are enabled by replacing the journalist with what audiences experience as an unpretentious, optimistic and helpful journalistic persona that speaks their language and understands their needs. For the audience that uses it, the friendly newsbot contrasts with everything they previously thought journalists to be. The public service journalists who operate the bot are, in turn, using the bot to try to reach new audiences by experimenting with a more informal, intimate relationship with ABC users. The supposedly "intelligent" (but in actual fact very much human-crafted) newsbot is the vehicle through which this new relationship is being forged.

In other words, the change to newsbots is the result of the newsbot's design, its method of delivering news via modular dialogic elements that simulate a human conversation, and the ways in which the bot is perceived in terms of personality characteristics by its niche audience. No longer is the newsbot characterised by its ability to disseminate news to a single, monolithic audience. In the $\mathrm{ABC}$ newsbot we see the particular personality of the newsbot forging a trusted relationship with a niche audience that previously did not engage with the news, via changes to the tone and authorial voice of the journalistic persona delivering it.

The $\mathrm{ABC}$ newsbot team recognised early on that this audience was dissatisfied with news that they perceived as depressing, and that they were intimidated by what the news expected from users. The newsbot team crafted a personality for a bot that is able to achieve 
what journalists may not be able to achieve as individual professionals. The bot is able to fill in gaps in basic knowledge, provide explainers, and insert colloquialisms through the use of humour and satire, while imagining what the audience might say about the news when they share it among friends.

For the $\mathrm{ABC}$, the news chatbot enables them to perform their public information function for a target audience that did not previously consume news, pushing messages about important public issues and events (about the national budget, about the same sex marriage referendum, about natural disasters in the area) in a way that provide an opportunity to be more readily viewed and talked about. The $\mathrm{ABC}$ newsbot, then, fulfils important universality goals for the organisation, potentially bringing new audiences to the public domain. It forges new connections with audiences by re-charting public space. Instead of multiple ways of broadcasting the same news to an audience that gathers in the PSM sphere, these newsbots are comprised of a collection of intimate conversations conducted within the more personal space of the messaging environment. In this way, automation and personalisation features serve to enhance trust in the $\mathrm{ABC}$ - at least among those who use the newsbot.

It is important to recognise, however, that the positive consequences for universality and trust that we currently see in this newsbot are subject to complex relations embedded in the delivery of the newsbot service. Although the ABC focuses a significant amount of attention on its journalistic efforts on third-party platforms such as Facebook, Twitter and YouTube (Levy 2017; Mason 2016; ABC 2017) and sees their connection with third-party providers as a way of advancing universality, this undoubtedly has consequences for the PSM accountability principles. In this case, the $\mathrm{ABC}$ is dependent on the private infrastructure of Facebook and ChatFuel. This raises issues relating to data ownership, the tracking and privacy of citizens and audiences' perceptions about the trustworthiness of the companies operating private messaging environments (Lokot and Diakopoulos 2015; Guzman 2017). Such matters will only become more relevant as news is delivered increasingly via smart speakers and controlled by the few global corporations with the expertise, data and resources necessary for leading such developments.

Understanding how public service media organisations are engaging with automation through partnerships with private companies and the effects of such engagement on the relationship between citizens and their public service media organisation is critical in order to ensure that innovations by public service bodies remain closely aligned with the values and goals that they aspire to. Our study the importance of the public's response to novel news formats - particularly in relation to conversational journalism and personalised news. Recognising and respecting the attitudes of citizens who have previously been left out of news consumption is critical in evaluating the democratic value of such developments. But further research is necessary in order to understand the significance of newsbots in terms of the developing relationship between the public and their public service media, particularly as the realm of smart speaker technology expands.

How audiences receive and evaluate knowledge claims made using automated processes is important because the ways in which audiences receive data-generated news potentially change the relationship between news producers and the publics they serve. And that relationship is a worthy area of study.

\section{References}

ABC. 2016. "ABC Annual Report, 2016." ABC. 21 February. http://about.abc.net.au/wpcontent/uploads/2016/11/ABCAnnualReport2016.pdf

ABC. 2017. "The ABC on Third Party Sites." ABC. 2 December. http://about.abc.net.au/wpcontent/uploads/2016/07/ABCThirdPartySitesGDE.pdf. 
Bernard, Travis. 2016. "Check out the new AI-powered TechCrunch news bot on Telegram Messenger." TechCrunch (blog). March 15. https://techcrunch.com/2016/03/15/check-out-thenew-ai-powered-techcrunch-news-bot-on-telegram-messenger/.

Born, Georgina. 2005. Uncertain Vision: Birt, Dyke and the Reinvention of the BBC. London: Random House.

Bradshaw, Paul. 2016. “2016 Was the Year of the Bot - Here's a Brief History of How They Have Been Used in Journalism." Online Journalism Blog. December 21.

https://onlinejournalismblog.com/2016/12/21/how-bots-came-to-play-a-role-in-journalisma-brief-history/.

Debrett, Mary. 2010. Reinventing Public Service Television for the Digital Future. Bristol: Intellect.

Edelman. 2018. "2018 Edelman Trust Barometer." https://www.edelman.com/trust-barometer.

Flew, Terry. 2011. "Rethinking Public Service Media and Citizenship: Digital strategies for news and current affairs at Australia's Special Broadcasting Service (SBS)." International Journal of Communication 5 (2011): 215-232. doi: 1932-8036/20110215.

Gouritin, Thomas. 2017. "Let's agree to stop it with the AI chatbot bullshit in 2018, k?" The Next Web, December 22. https://thenextweb.com/contributors/2017/12/21/lets-agree-stop-aichatbot-bullshit-2018-k.

Guzman, Andrea L. 2017. "Making AI safe for humans: A conversation with Siri.” In Robert W. Gehl \& Maria Bakardjieva (Eds.), Socialbots and their friends: Digital media and the automation of sociality. London: Routledge.

Höflich, Joachim R. 2013. "Relationships to Social Robots: Towards a Triadic Analysis of Mediaoriented Behavior." Intervalla: Platform for Intellectual Exchange 1 (1):35-35.

Jakubowicz, Karol. 2007. "Public Service Broadcasting: A Pawn on an Ideological Chessboard." In Media Between Culture and Commerce, edited by Els de Bens, 115-150. Chicago: Intellect.

Levy, David. 2017. "The ABC: A Case Study in Updating PSB in Politically Polarised and CashStrapped Times." White Paper. Oxford: Reuters Institute for the Study of Journalism. http://reutersinstitute.politics.ox.ac.uk/sites/default/files/2017-07/Levy\%20\%20The\%20ABC.pdf

Light, Ben, Jean Burgess, and Stefanie Duguay. 2018. "The Walkthrough Method: An Approach to the Study of Apps." New Media \& Society 20 (3): 881-900. doi: 0.1177/1461444816675438.

Lokot, Tetyana, and Nicholas Diakopoulos. 2015. "News Bots: Automating news and information dissemination on Twitter." Digital Journalism 4 (6): 682-699. doi: 10.1080/21670811.2015.1081822.

Marsh, Peter. 2017. "Backstory: Getting personal on Facebook Messenger." ABC News Online. 7 October. http://www.abc.net.au/news/about/backstory/digital/2017-10-07/abc-newsgetting-personal-in-messenger/8987354

Mason, Max. 2016. "ABC's Michelle Guthrie says Netflix deal allows for quality programming." Financial Review, 16 November. https://www.afr.com/business/media-and-marketing/tv/abcmd-michelle-guthrie-says-netflix-deal-allows-for-quality-programming-20161116-gsqoe 4 
McClean, Georgia. 2011. "Multicultural Sociability, Imperfect Forums and Online Participation." International Journal of Communication 5: 1649-1668. doi: 1932-8036/20111649.

Moon, Youngme, \& Nass, Clifford. 1996. "How "Real” Are Computer Personalities?: Psychological Responses to Personality Types in Human-Computer Interaction." Communication Research 23 (6), 651-674. doi: 10.1177/009365096023006002.

Nagy, Peter, and Gina Neff. 2015. "Imagined affordances: Reconstructing a keyword for communication theory." Social Media + Society 1 (2), 1-9. doi: $10.1177 / 2056305115603385$.

Newman, Nic, Richard Fletcher, Antonis Kalogeropoulos, David AL Levy, and Rasmus Kleis Nielsen. 2017. Reuters Institute Digital News Report 2018. Reuters Institute for the Study of Journalism.

Newman, Nic, Richard Fletcher, Antonis Kalogeropoulos, David AL Levy, and Rasmus Kleis Nielsen. 2018. Reuters Institute Digital News Report 2018. Reuters Institute for the Study of Journalism.

Pink, Sarah, Heather Horst, John Postill, Larissa Hjorth, Tania Lewis, and Jo Tacchi. 2015. Digital Ethnography: Principles and Practice. New York: SAGE.

Seaver, Nick. 2017. "Algorithms as culture: Some tactics for the ethnography of algorithmic systems." Big Data \& Society Advance online publication: 1-12. doi: $10.1177 / 2053951717738104$.

Sundar, S. Shyam, and Clifford Nass. 2001. "Conceptualizing Sources in Online News." Journal of Communication 51 (1), 52-72. doi: 10.1111/j.1460-2466.2001.tb02872.x

Thurman, Neil, Judith Moeller, Natali Helberger, and Damian Trilling. 2018. "My Friends, Editors, Algorithms, and I." Digital Journalism, Advance online publication. doi: 10.1080/21670811.2018.1493936.

Tolliday, Rob. 2017. Sensis Social Media Report 2017. Sydney Sensis. October 282018. https://www.sensis.com.au/about/our-reports/sensis-social-media-report

Tracey, Michael. 1998. "Principles of Public Service Broadcasting.” Chap. 3 in Decline and Fall of Public Service Broadcasting. London: Oxford University Press.

Van de Bulck, Hilde, and Halvard Moe. 2017. "Public Service Media, Universality and Personalisation Through Algorithms: Mapping strategies and exploring dilemmas." Media, Culture \& Society 40 (6): 875-892. doi: 10.1177/0163443717734407.

Van Dijck, Jose, \& Thomas Poell. 2015. “Making Public Television Social? Public Service Broadcasting and the Challenges of Social Media." Television and New Media 16 (2): 148-164. doi: $10.1177 / 1527476414527136$.

Verma, Vaibhav. 2018. "This 50-Time Chatbot Designer Shares the Basics of Creating Engaging Chatbots." Tech in Asia, February 9, 2018. https://www.techinasia.com/talk/50-time-chatbotdesigner-basics-engaging-chatbots. 


\section{Figures Captions}

Figure 1. An example of an $\mathrm{ABC}$ News chatbot morning news alert

Figure 2. The ABC newsbot's response to our question: "What is trending on social media?" Figure 3. Users are provided with an "Explainer" for the news headlines

\footnotetext{
${ }^{1}$ Letters and numbers refer to our numbering scheme for coding anonymised responses.

${ }^{2}$ See http://knowyourmeme.com/memes/shut-up-and-take-my-money
} 